\title{
Understanding B Meson Branching Fractions
}

\section{Bernard Gittelman}

October 10, 2001

\begin{abstract}
This is a discussion of the main branching fractions of B Meson decays $\left(b \rightarrow c W^{-}\right)$. What has been measured and what remains unmeasured is summarized, and a plan to measure charged track multiplicity accompanying the $D^{(*)}$ meson in B decay is presented.
\end{abstract}

\section{Introduction}

The existence of the B Meson was infered from the discovery of the $\Upsilon(1 S)$ and $\Upsilon(2 S)$ mesons at Fermilab in 1977[1], and the measurement of the electromagnetic production cross section, $e^{+} e^{-} \rightarrow \Upsilon(1 S)$ and $e^{+} e^{-} \rightarrow \Upsilon(2 S)$, in 1978 at the DORIS storage ring of DESY[2]. From the magnitude of these cross-sections, it became clear that the $\Upsilon$ resonances are to be described as $b \bar{b}$ quark pairs, not $t \bar{t}$. The Cornell-Electron-Storage-Ring, CESR, started operating in late 1979, and the two Collaborations there, CLEO and CUSB published observation of the $\Upsilon(1 S), \Upsilon(2 S)$, $\Upsilon(3 S)[3]$, and $\Upsilon(4 S)[4]$ in 1980 . The $\Upsilon(4 S)$ is a much wider resonance and is believed to decay exclusively to $B \bar{B}$ mesons. Over the past 20 years, the general properties of the $B$ meson decays have been studied by many different groups[5]. For reasons having to do with minimizing backgrounds, CLEO has studied B mesons by taking data at the $\Upsilon(4 S)$. For every data sample recorded at the $\Upsilon(4 S)$, CLEO has collected a data sample of approximately half as much integrated luminosity at an energy of 40 to $80 \mathrm{MeV}$ below the $\Upsilon(4 S)$ to measure the background from the continuum. This energy is below $B \bar{B}$ threshold. The original CLEO detector, CLEO-I, was used from 1979 until 1988. It was then replaced by the CLEO-II detector, which consisted of a system of tracking chambers with much better resolution, and a Cesium-Iodide crystal electromagnetic calorimeter (see figure 1). In 1995, the CLEO-II detector was upgraded, by replacing the inner-most tracking chamber, (the PTL), with a silicon vertex tracker. The newer version of CLEO-II was named CLEO-II.V. In 1998, CESR shut down for upgrading, and the CLEO-II.V detector was upgraded further and renamed CLEO-III. CLEO-III contains a new drift chamber, a new silicon detector, and a Ring-Imaging-CHerenkov detector, RICH, for better pion-kaon identification, but the time-of-flight scintillation counters were removed. Data taking with CLEO-III began in 2000. Figures 1 and 2 illustrate the CLEO-II and CLEO-III detectors. Table 1 provides a summary of the integrated luminosity recorded on the $\Upsilon(4 S)$ for each of the CLEO detectors.

Lecture presented at the Hadron-2001 Conference, 26-Aug-2001; Protvino, Russia 


\section{Inclusive Branching Fractions}

B meson decay is described by 4 basic diagrams, as shown in Fig. 3. The diagrams describing $b \rightarrow c W^{-}$and $b \rightarrow u W^{-}$are shown in Figs $3 \mathrm{a}$ and $3 \mathrm{~b}$. The magnitude of $b \rightarrow c W^{-}$branching fractions versus $b \rightarrow u W^{-}$branching fractions have been estimated by the study of inclusive production of $D$ mesons in nonleptonic and semi-leptonic final states. Evidence for the existence of diagrams 3c and $3 \mathrm{~d}$ (the penquins), comes from the observation of $b \rightarrow s \gamma$ final states such as $B \rightarrow K^{*} \gamma$. An estimate of the importance of the different contributions to B meson decays as measured from the inclusive branching fractions is given in Table 2.

\section{Semi-leptonic Decay}

The existence of the B meson was first verified by the observation of leptons (electrons and muons) coming from $\Upsilon(4 S)[6]$ decays, with a momentum spectrum expected from $B$ meson semi-leptonic decays $[6]$. The semi-leptonic decay of $B$ mesons has been studied in detail. A summary of what is known today about the branching fractions is given in Table 3[7]. The total branching fraction for semi-leptonic decay is approximately $24 \%$. This comes from the almost equal branching fraction for inclusive electrons and muons, and an estimate of the expected branching fraction for $\tau$ leptons using the phase space factor. As shown in Table 3, some of the exclusive final state branching fractions have been measured. The sum of the measured exclusive semi-leptonic branching fractions is approximately $72 \%$ of the inclusive semi-leptonic branching fraction. From the fact that the $b \rightarrow u W^{-}$semileptonic final state branching fractions are approximately 100 times smaller than the $b \rightarrow c W^{-}$states, we estimated the total inclusive $b \rightarrow u W^{-}$branching fraction shown in Table 2.

\section{Hadronic Final States}

From inclusive measurements of $B \rightarrow D X, 87 \% ; B \rightarrow \Lambda_{c} X, \approx 6 \%$; and $B \rightarrow$ $(c \bar{c}) X, \approx 2 \%$; one concludes that $b \rightarrow c W^{-}$is responsible for most, (95\%), of $\mathrm{B}$ decays. The challenging problem is to measure the exclusive branching fractions. The measured two body exclusive branching fractions for hadronic decay of $\mathrm{B}$ mesons are listed in Table 4. One observes for $B \rightarrow D^{(*)} X$, total measured for $B^{-}=10 \%$, and for $B^{\circ}=6.9 \%$; for $B \rightarrow(c \bar{c}) X$, total measured is $0.41 \%$ and $0.33 \%$; for $b \rightarrow$ $u W^{-}$plus $b \rightarrow s g$ plus $b \rightarrow s \gamma$, the total branching fractions are $0.017 \%$ and $0.012 \%$. This again leads to the conclusion, understanding the $b \rightarrow c W^{-}$decays will enable us to describe most (more than $90 \%$ ) of $B$ meson decays. The measured, three, four, and five body final state branching fractions for the hadronic decay of $\mathrm{B}$ mesons via $b \rightarrow c W^{-}$are listed in Table 5 . The measured exclusive branching fractions for 2 body final states is much larger than for 3,4 , or 5 body states, but that probably has to do with complexity and larger combinatoric backgrounds for multi-body $(>2)$ final states. We know of no reason to expect the total branching fraction for 3 body, 4 body or 5 body hadronic states $\left(D^{(*)}\right.$ meson plus 4 particles) should be so much 
smaller than that of 2 bodies states.

\section{A Proposed Method to measure "Exclusive" B Decays.}

From the decay modes listed in Table 3, the total exclusive semi-leptonic branching fractions that have been measured is $7.5 \%$; i.e., we have measured $7.5 / 10.5=$ $72 \%$ of the semi-leptonic decays. Adding up the various hadronic decay modes listed in Tables 4 and 5 and averaging over $B^{-}$and $B^{\circ}$, we find only $14.8 \%$ for the hadronic final states branching fractions. A summary of the major hadronic decay branching fractions listed by multiplicity is given in Table 6. Assuming the total hadronic branching fraction is $(100-24) \%=76 \%$, we have measured only $14.8 / 76$ $=19 \%$ of the exclusive hadronic decay modes. It is interesting to speculate on why we have measured $72 \%$ of the semi-leptonic final states where we are always missing the energy and momentum of the neutrino, but only measured $19 \%$ of the hadronic final states where for most modes we should be able to detect every final state particle (photons, charged pions, kaons, and protons, but not neutrons). The only explanation is based on higher multiplicity of the hadronic final state.

As mentioned earlier, CLEO has collected approximately $15 \mathrm{fb}^{-1}$ of data on the $\Upsilon(4 S)$ resonance. This provides $\sim 15 \times 10^{6} B \bar{B}$ pairs. If one were to try reconstructing the $B$ meson in one of the many clean easy to reconstruct decay modes, and the $\mathrm{D}$ (or $\Psi$ meson) from the $\bar{B}$ decay in the same event, then one would have a very useful sample of $\bar{B}$ decays. In this reconstruction process, we will be removing events in which the sum of the charge of the tracks does not add up to zero, and those events in which the total momentum vector of the $\mathrm{D}$ meson, plus the charged tracks, and the photons does not point in "exactly" the direction opposite to the momentum vector of the reconstructed $B$ meson. We have estimated having 15,000 events in which we have reconstructed The $B$ meson and the $D$ meson from the $\bar{B}$ decay. This sample of 15,000 events might be useful to let us know how often the $\mathrm{B}$ meson decays into

$$
\begin{gathered}
B \rightarrow D+1 \text { track } ; D+2 \text { tracks } ; \ldots ; D+9 \text { tracks } ; \ldots \\
B \rightarrow D+\pi^{o} ; D+\eta^{o} ; D+\pi^{o}+1 \text { track } ; D+\eta^{o}+1 \text { track } ; \ldots ; D+\eta^{o}+9 \text { tracks } ; \ldots
\end{gathered}
$$

Perhaps we will also be able to learn how often the B meson decays into

$$
\begin{gathered}
B \rightarrow D^{*}+\text { ntracks } ; \quad D^{*}+\pi^{o}+\text { ntracks } ; D^{*}+\eta^{o}+\text { ntracks } \\
B \rightarrow D^{(*)}+2 \pi^{o}+\text { ntracks } ; \quad D^{(*)}+3 \pi^{o}+\text { ntracks }
\end{gathered}
$$

Given a sample of $B \rightarrow D^{(*)}+n$ tracks, one can investigate the invariant mass distribution of the $n$ tracks. We would also study the invariant mass distribution of all two track combinations for each set of n charged tracks accompanying the $D^{(*)}$ mesons. 


\section{Summary}

Evidence for B mesons was discovered in 1980 and after 20 years, the decay modes are understood at the $40 \%$ level (See the last line of Table 6.). The D mesons were discovered in 1975 , but $90 \%$ of their decay modes are already measured. This enormous difference is due to the three times higher mass of the B meason leading to more complicated, higher multiplicity states. However, we know from inclusive measurements that more than $90 \%$ of $\mathrm{B}$ mesons decay to a $D X(87.6 \%)$, $\Psi X(1.15 \%)$, or $\Lambda X(6.4 \%)$. It will be interesting if we are able to measure the charged track multiplicity of the $X$ part of $B \rightarrow D X$ final states, to learn whether the Standard Model with QCD is able to describe (predict) these multiplicities. Measuring the same for $B \rightarrow D^{*} X$ (and also for $B \rightarrow D \pi^{\circ} X$ and $B \rightarrow D^{*} \pi^{o} X$ ) would be very useful. If our $15 \mathrm{fb}^{-1}$ data sample is large enough (?), we should be able to complete this over the next two years.

\section{$\underline{\text { References }}$}

1. Observation of a new group of resonances at $10 \mathrm{GeV}$ decaying to muons pairs

S.W. Herb et al., Phys. Rev. Lett. $\underline{39}, 252(1977)$.

2. Confirmation of the existence of the $\Upsilon(1 S)$ and $\Upsilon(2 S)$

C. Berger et al., Phys. Lett. $\underline{76 B}, 243(1978)$.

C.W. Darden et al., Phys. Lett. $\underline{78 B}, 246(1978)$.

J.K. Bienlein et al., Phys. Lett. $\underline{78 B}, 360(1978)$.

C.W. Darden et al., Phys. Lett. $\underline{80 B}, 419(1979)$.

3. Observation of Three Upsilon States

D. Andrews et al., Phys. Rev. Lett. 44, 1108(1980).

T. Bohringer et al., Phys. Rev. Lett. $\underline{44}, 1111(1980)$.

4. Observation of a Fourth Upsilon State in $e^{+} e^{-}$Annihilations

D. Andrews et al., Phys. Rev. Lett. $\underline{45}, 219(1980)$.

G. Finnocchiaro et al., Phys. Rev. Lett. $\underline{45}, 222(1980)$.

5. Collaborations studying $B$ meson decays:

CLEO, ARGUS, ALEPH, DELPHI, OPAL, SLD, CDF

6. M.E.Nelson et al., Phys. Rev. Lett. 50, 1542(1983).

E.Fernandaz et al., Phys. Rev. Lett. $\underline{50}, 2054(1983)$.

7. The branching fractions quoted in this paper are approximate values. Most of these numbers have been checked for consistency with the Review of Particle Physics, The European Physical Journal, 15, 1-4(2000). 
Table 1, The CLEO Detectors Used to Study B Meson Decay

CLEO has been studying B Decay since 1980

Detector

CLEO_I

CLEO_II

CLEO_II.V

CLEO_III
$\Upsilon \mathbf{\Upsilon}(4 \mathbf{S})$ Luminosity

$$
\begin{array}{rr}
1980-1989 & 295 p b^{-1} \\
1990-1995 & 3136 p b^{-1} \\
1996-1998 & 6064 p b^{-1} \\
2000-2001 & \sim 6000 p b^{-1}
\end{array}
$$

Table 2, Status of Our understanding of B Meson Decays

$$
\begin{array}{ll}
b \rightarrow c W^{-} & \sim(92-95) \% \\
b \rightarrow u W^{-} & \sim(1.2-2) \% \\
b \rightarrow s g & \quad ? ? \\
b \rightarrow s \gamma & \sim\left(3 \times \mathbf{1 0}^{-2}\right) \%
\end{array}
$$

Table 3, B Meson Semi-Leptonic Decays $\quad \bar{B} \rightarrow l^{-} \bar{\nu}_{l} X$

(From inclusive measurements of Semi-Leptonic Decays)

The leptons, $l$,

$$
\begin{array}{ll}
l^{-}=e^{-} & 10.5 \% \\
l^{-}=\mu^{-} & 10.4 \% \\
l^{-}=\tau^{-} & \sim 3 \% \text { (from theory) }
\end{array}
$$

Total 'measured' $10.5+10.4+3.0=24 \%$

X, the hadronics, for $B^{-} \quad \bar{B}^{o}$

\begin{tabular}{lll}
$X=D$ & $\mathbf{2 . 1 5 \%}$ & $\mathbf{2 . 1 0 \%}$ \\
$X=D^{*}$ & $\mathbf{5 . 3 \%}$ & $\mathbf{4 . 6 \%}$ \\
$X=D^{* *}$ & $\mathbf{0 . 6 \%}$ & $\mathbf{?}$ \\
& \multicolumn{2}{l}{} \\
$X=\pi$ & $? ?$ & $\left(1.8 \times \mathbf{1 0}^{-2}\right) \%$ \\
$X=\rho$ & $? ?$ & $\left(2.6 \times \mathbf{1 0}^{-2}\right) \%$ \\
--------- & \\
Total measured & $\sim 8 \%$ & $\sim 7 \%$
\end{tabular}


Table 4, Two Body B Meson Hadronic Decays

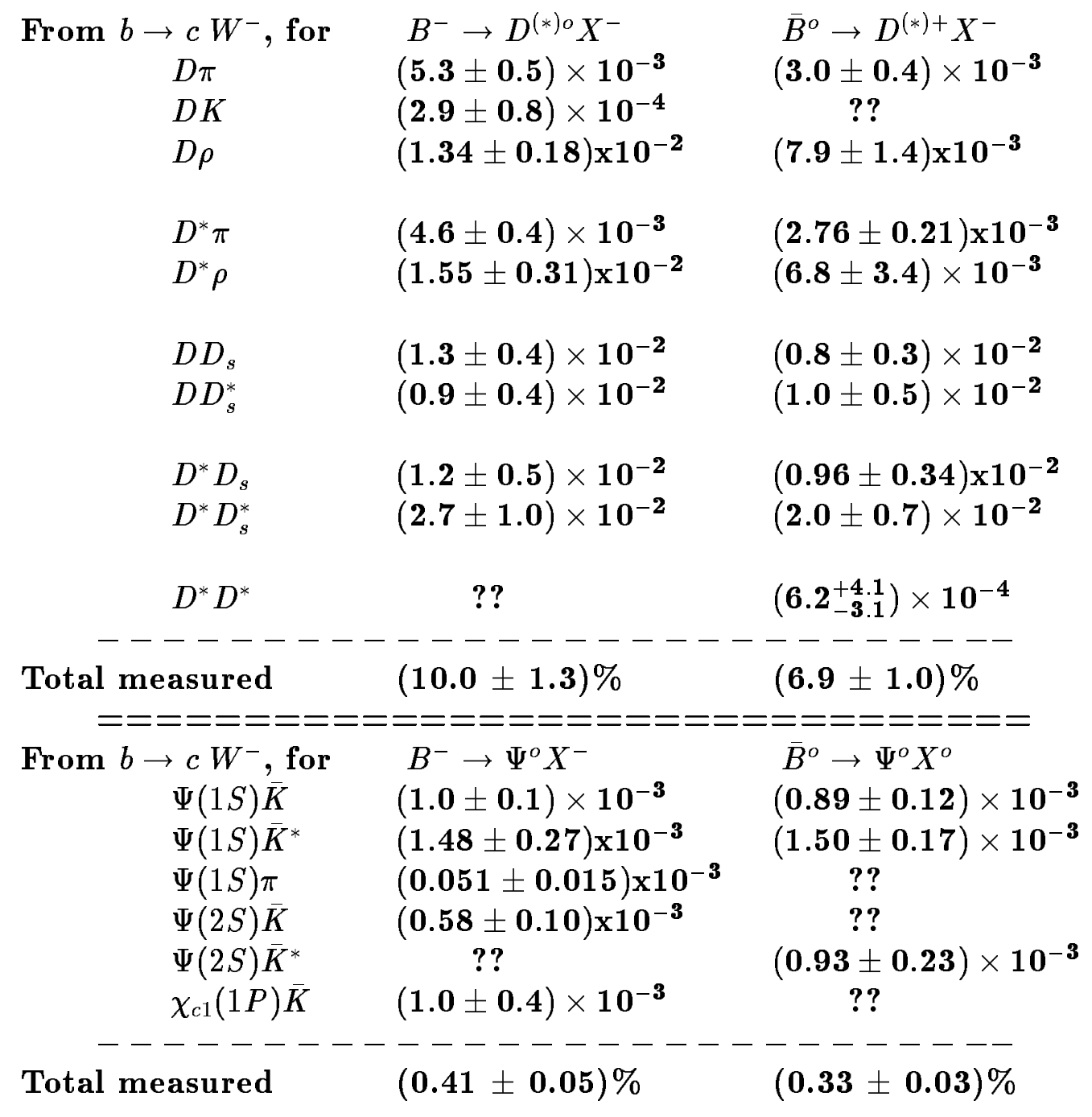

From $b \rightarrow u W^{-}$and /or $b \rightarrow s g$

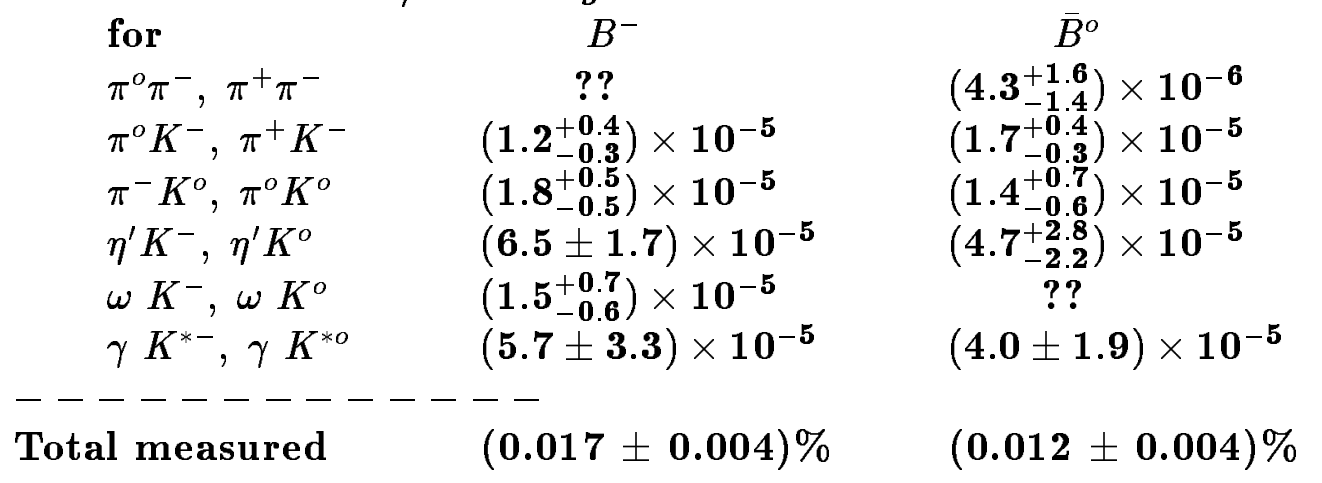


Table 5, Multi-Body B Meson Hadronic Decays

Three Body Hadronic Final States
From $b \rightarrow c W^{-}$, for$$
B^{-} \rightarrow D^{(*)} X
$$
$D^{o} \pi^{-} \rho^{o} \quad(4.2 \pm 3.0) \times 10^{-3}$
??
$D^{* o} \pi^{-} \pi^{o}$
$D^{*+} \pi^{-} \pi^{-}$
$D^{+} \pi^{-} \rho^{o} \quad(1.1 \pm \mathbf{1 . 0}) \times 10^{-3}$
$D^{*+} \pi^{-} \pi^{o}$
$D^{* o} \pi^{+} \pi^{-}$
$(1.5 \pm 0.5) \times 10^{-2}$ ??
$(\mathbf{2 . 1} \pm \mathbf{0 . 6}) \times \mathbf{1 0}^{-3}$
$\bar{B}^{o} \rightarrow D^{(*)} X$
$\Psi(1 S) K^{-} \pi^{o}$

$$
B^{-} \rightarrow \Psi X^{-}
$$
$\bar{B}^{o} \rightarrow \Psi X^{o}$
$\Psi(1 S) K^{-} \pi^{+} \quad(\mathbf{1 . 2} \pm \mathbf{0 . 6}) \times \mathbf{1 0}^{-\mathbf{3}}$
$\begin{array}{ll}B^{-} \rightarrow N \bar{N}^{\prime} X \\ \Lambda_{c}^{+} \bar{P} \pi^{-} & (\mathbf{6 . 2} \pm \mathbf{2 . 7}) \times \mathbf{1 0}^{-4}\end{array}$
$\begin{array}{cc}\Lambda_{c}^{+} \bar{N} \pi^{-} & \bar{B}^{o} \rightarrow N \bar{N}^{\prime} X \\ ? ?\end{array}$

Total measured $\quad(0.69 \pm 0.31) \%$

$(1.7 \pm \mathbf{0 . 5}) \%$

$=====================$

Four Body Hadronic Final States
From $\mathbf{b} \rightarrow \mathbf{c ~ W}^{-}$, for

$$
B^{-} \rightarrow D^{(*)} X
$$
$D^{\circ} \pi^{-} \pi^{-} \pi^{+} \quad(5 \pm 4) \times 10^{-3}$
$D^{* o} \pi^{-} \pi^{-} \pi^{+}$
$(9.4 \pm 2.6) \times 10^{-3}$
$D^{*+} \pi^{-} \pi^{-} \pi^{o}$
$(1.5 \pm 0.7) \times 10^{-2}$
$\bar{B}^{o} \rightarrow D^{(*)} X$
$D^{*+} \pi^{-} \pi^{-} \pi^{+}$
$D^{\star o} \pi^{-} \pi^{+} \pi^{o}$
$D^{+} \pi^{-} \pi^{-} \pi^{+} \quad(\mathbf{8 . 0} \pm \mathbf{2 . 5}) \times \mathbf{1 0}^{-3}$
$(6.8 \pm 3.4) \times 10^{-3}$
$B^{-} \rightarrow \Psi X^{-}$
$\Psi(1 S) K^{-} \pi^{+} \pi^{-}(\mathbf{1 . 4} \pm \mathbf{0 . 6}) \times \mathbf{1 0}^{-\mathbf{3}}$
$\Psi(2 S) K^{-} \pi^{+} \pi^{-}(\mathbf{1 . 9} \pm \mathbf{1 . 2}) \times \mathbf{1 0}^{-3}$
$\begin{array}{ll}\Psi(1 S) K^{-} \pi^{+} \pi^{o} & ? ? \\ \Psi(1 S) K^{-} \pi^{+} \pi^{o} & ? ?\end{array}$
$B^{-} \rightarrow N \bar{N}^{\prime} X$
$\Lambda_{c}^{+} \bar{P} \pi^{-} \pi^{o}$
??
$\bar{B}^{o} \rightarrow N \bar{N}^{\prime} X$
measured
Total measured $\quad(3.3 \pm 0.9) \%$
$\Lambda_{c}^{+} \bar{P} \pi^{+} \pi^{-} \quad(\mathbf{1 . 3} \pm \mathbf{0 . 6}) \times \mathbf{1 0}^{-3}$

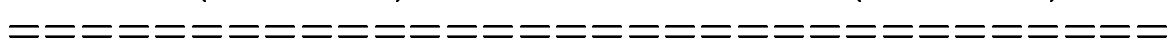

Five Body Hadronic Final States

From b $\rightarrow \mathbf{c ~ W}^{-}$, for

$$
B^{-} \rightarrow D^{(*)} X
$$

$D^{\circ} \pi^{-} \pi^{-} \pi^{+} \pi^{o} \quad(0.41 \pm 0.09) \%$

$D^{* o} \pi^{-} \pi^{-} \pi^{+} \pi^{o} \quad(1.8 \pm 0.4) \%$

$$
\begin{gathered}
\bar{B}^{o} \rightarrow D^{(*)} X \\
D^{+} \pi^{-} \pi^{-} \pi^{+} \pi^{o}(\mathbf{0 . 2 8} \pm \mathbf{0 . 0 6}) \% \\
D^{*+} \pi^{-} \pi^{-} \pi^{+} \pi^{o}(\mathbf{1 . 7 2} \pm \mathbf{0 . 2 8}) \% \\
D^{* o} \pi^{-} \pi^{+} \pi^{-} \pi^{+}(\mathbf{0 . 3} \pm \mathbf{0 . 1}) \%
\end{gathered}
$$

$D^{* o} \pi^{-} \pi^{-} \pi^{+} \pi^{o}$

??

Total measured $\quad(2.2 \pm 0.4) \%$

$(\mathbf{2 . 3} \pm \mathbf{0 . 3}) \%$ 


\title{
Table 6, B Meson Branching Fractions
}

\author{
$\mathbf{B}^{-}$ \\ $\overline{\mathrm{B}}^{\mathbf{o}}$
}

Inclusive Semi-Leptonic

\begin{tabular}{lll}
\hline electrons, $\mathbf{e}^{-}$ & $\mathbf{( 1 0 . 9} \pm \mathbf{0 . 6}) \%$ & $(\mathbf{1 0 . 2} \pm \mathbf{0 . 6}) \%$ \\
muons, $\mu^{-}$ & $\mathbf{( 1 0 . 8} \pm \mathbf{0 . 6}) \%$ & $\mathbf{( 1 0 . 1} \pm \mathbf{0 . 6}) \%$ \\
tau, $\tau^{-}$ & $\mathbf{( 3 . 1} \pm \mathbf{0 . 6}) \%$ & $\mathbf{( 2 . 9} \pm \mathbf{0 . 6 ) \%}$ \\
& ------ & ------ \\
& $\mathbf{( 2 4 . 7} \pm \mathbf{1 . 1}) \%$ & $\mathbf{( 2 3 . 1} \pm \mathbf{1 . 1}) \%$
\end{tabular}

Two Body Hadronic Final States with $D$ or $D^{*}$ or $D^{* *}$

$(10.0 \pm 1.3) \% \quad(6.9 \pm 1.0) \%$

Two Body Hadronic Final States with $c \bar{c}$. (i.e., $\Psi$ 's)

$(0.41 \pm 0.05) \% \quad(0.33 \pm 0.03) \%$

Three Body Hadronic Final States

$$
\mathbf{( 0 . 6 9} \pm \mathbf{0 . 3 1}) \% \quad(1.7 \pm 0.5) \%
$$

Four Body Hadronic Final States

$$
(3.3 \pm 0.8) \% \quad(1.6 \pm 0.4) \%
$$

Five Body Hadronic Final States

$$
(\mathbf{2 . 2} \pm \mathbf{0 . 4 ) \%} \quad(\mathbf{2 . 3} \pm \mathbf{0 . 3}) \%
$$

Total, $b \rightarrow c$, Inclusive Semi-Leptonic Decays plus

Exclusive Hadronics Decays

$$
(41.3 \pm 1.8) \% \quad(35.9 \pm 1.7) \%
$$

